\title{
Evidence for Nonvanishing Cosmological Constant in NonSusy Superstring Models
}

\author{
Roberto Iengo* \\ Scuola Internazionale Superiore di Studi Avanzati (SISSA) \\ and INFN, Sezione di Trieste \\ Via Beirut 4, I-34013 Trieste, Italy \\ Chuan-Jie Zhu ${ }^{\dagger}$ \\ Institute of Theoretical Physics, Chinese Academy of \\ Sciences, P. O. Box 2735, Beijing 100080, P. R. China \\ May 7, 2018
}

\begin{abstract}
We reanalyse the computation of the cosmological constant $\Lambda$ at two loops in recently proposed Superstring models without massless gravitini, both in the theta-function based formalism and by a detailed computation in the more explicit hyperelliptic description of the underlying genus two Riemann surface. $\Lambda$ is expressed as the integral over the surface moduli of an amplitude which is zero if susy is not completely broken, but we find it to be nonvanishing in the susy breaking models which can be given an explicitly workable fermionic formulation. Thus unfortunately the issue of getting realistic and perturbatively viable models from Superstring Theory remains open.
\end{abstract}

*E-mail: iengo@he.sissa.it

${ }^{\dagger}$ E-mail: zhucj@itp.ac.cn 


\section{Introduction and summary}

The vanishing of the cosmological constant $\Lambda$ is a crucial issue in Superstring theory. In fact, if $\Lambda$ is nonzero the theory is perturbatively unstable. Unavoidable (infrared) divergences appear due to the dilaton tadpole (proportional to $\Lambda$ ), physically signaling the fact that the flat background, over which the perturbative expansion is performed, is no longer consistent. Therefore also the perturbative spectrum and thus the particle content of the theory is no longer trusty, and its physical meaning becomes (at least so far) inaccessible.

The standard versions of Superstring theory have indeed zero cosmological constant [1, 2]. This can be formally argued as a consequence of exact supersymmetry, and it has been explicitly proven at one and two loops [3], both for the maximally supersymmetric models and also for less (but still at least $N=1$ ) supersymmetry [4]. It is therefore very important to investigate whether it is possible to construct more realistic string theories, where there are no massless gravitini and supersymmetry is completely broken. Models of that kind have recently appeared in the literature [5, 6], and including arguments to show that $\Lambda$ is still zero [7]. Some of those models can be given an explicitly workable fermionic formulation [6], by using which it is possible to make a detailed analysis also at two loops. Note that the one and two loop computations are physically very different. In fact at one loop one evaluates the partition function of a free theory, whereas only at two loops one begins to see the effect of the interaction.

Here we reanalyse those models. Our rather explicit computation will make use of the very convenient hyperelliptic formalism which provides a complete description of the genus two Riemann surface. Thus it looks different from the formalism used in the argument appeared in the recent literature about the two loops vanishing of the $\Lambda$ in the nonsusy models. However we have also reanalysed those arguments [6, 7] and found some flaws. One of the main ingredients of the two loop computation in any formalism is the occurrence in the amplitude of a correlator of supercurrents, related to the fact that at genus two there are superghost zero modes also for the even spin structures. Now, in some arguments this correlator is ignored [6] (note that the correlator is sigular at coincident points); if one does so the result is

indeed zero (as we check in our explicit computation, see eq. (68)). However the amplitude is not the right one. 
In more refined arguments this correlator is included. Let us see it more precisely (see for instance [8]). The amplitude $A$, to be integrated over the moduli of the surface, is obtained by evaluating the expectation value of the following quantity (times determinants of some differential operators):

$$
\delta\left(\beta\left(z_{1}\right)\right) \psi^{\mu} \partial X_{\mu}\left(z_{1}\right) \delta\left(\beta\left(z_{2}\right)\right) \psi^{\mu} \partial X_{\mu}\left(z_{2}\right) .
$$

This is eq. (2.10) of [8], taking the supercurrent insertion in a point: $\chi_{a}=$ $\delta\left(z_{a}\right)$, and considering the matter part $J_{m}=\psi^{\mu} \partial X_{\mu}$ of the supercurrent. In the full expression one has to complete the supercurrent by adding the ghost part and the compactified matter part to the matter part, but for the purpose of the short discussion here it is enough to look at the partial expression above.

The result is (up to theta-function factors):

$$
A=(\text { theta-functions }) \frac{k_{i j} \omega_{i}\left(z_{1}\right) \omega_{j}\left(z_{2}\right)+q \partial_{z_{1}} \partial_{z_{2}} \log E\left(z_{1}, z_{2}\right)}{E\left(z_{1}, z_{2}\right)^{2} \sigma\left(z_{1}\right)^{2} \sigma\left(z_{2}\right)^{2}},
$$

where $k_{i j}$ and $q$ are constants. This is obtained by using the bosonization eqs. (3.15) and (3.30) of [8]:

$$
\left\langle\delta\left(\beta\left(z_{1}\right)\right) \delta\left(\beta\left(z_{2}\right)\right)\right\rangle=(\text { theta-functions }) / E\left(z_{1}, z_{2}\right) \sigma\left(z_{1}\right)^{2} \sigma\left(z_{2}\right)^{2},
$$

and by computing

$$
\left\langle J_{m}\left(z_{1}\right) J_{m}\left(z_{2}\right)\right\rangle=(\text { theta-functions }) \frac{\left(k_{i j} \omega_{i}\left(z_{1}\right) \omega_{i}\left(z_{2}\right)+q \partial_{z_{1}} \partial_{z_{2}} \log E\left(z_{1}, z_{2}\right)\right)}{E\left(z_{1}, z_{2}\right)} \text {. }
$$

Note that the amplitude $A$ has to be a 0 -differential in $z_{1,2}$, in order to be independent (up to irrelevant total derivatives in the moduli) on those arbitrary points. Remembering that $\sigma$ is a differential of degree equal to half the genus thus $\sigma^{2}$ is a 2 differential in our case (genus 2), that $E$ is a $-1 / 2$ differential and that $\omega$ like $\partial \log E$ is a 1-differential, we see that the result is in fact a 0 -differential in $z_{1,2}$, indeed the same 0 -degree as $\delta(\beta) \psi^{\mu} \partial X_{\mu}$.

Now, in eq. (5.1) of ref. [7] (the key point of that argument) the formula for $A$, eq. (2), appears with a crucial modification: that is, there is the extra factor $\operatorname{det}\left(\Phi_{a}^{3 / 2}\left(z_{b}\right)\right)$, where $\Phi_{a}^{3 / 2}$ are the superghost zero mode functions which are $3 / 2$-differentials. (Instead, the factor $1 / \sigma\left(z_{1}\right)^{2} \sigma\left(z_{2}\right)^{2}$ has not been written there, but since it is spin structure independent and nonzero for $z_{1} \rightarrow z_{2}$ it 
is not relevant for the ensuing argument). But actually there is not such a factor $\operatorname{det}\left(\Phi_{a}^{3 / 2}\left(z_{b}\right)\right)$. Indeed, as a check, since it is a half-integer differential, it does not match with the previously recalled property of $A$ to be a 0 differential. If we remove this factor to get the right amplitude, we see that the argument of ref. [7] does no longer show the vanishing of $\Lambda$, apart from possibly the "simpler model" of ref. [7] for which however the explicit form of the partition function and the explicit results for the spectrum have not been given, and the fundamental integration region over the moduli appears to be nonstandard.

This is confirmed by our explicit two loop computation for those models which can be given an explicitly workable free fermion formulation. First, in Sect. 2, we review the fermionic construction and the model (whose gravitini content is explicitly analysed in Appendix A) and in Sect. 3, in some details, the one loop computation showing that $\Lambda$ is zero at this order. The one loop computation indicates a convenient strategy for the two loop one. Accordingly, in Sect.4, the two loop amplitude is conveniently organized as a sum of various terms, which in Appendix B are classified as modular invariant sets. Many of those terms, Sect.4.1, are the same as for supersymmetric models and they were shown to vanish explicitly in ref. [4] (see also [9]), (we review this computation in the Appendix $\mathrm{C}$ following the strategy of one loop computation). Besides them there remain two sets of terms, which only occur in nonsusy models. Those of the first set, Sect.4.2, are shown to vanish by essentially the same kind of computation as at one loop (that is, showing that they contain at least one odd theta function). We finally, Sect.4.3, focus on the remaining set: here we find a nonvanishing result (the crucial expression is eq. (68), where we see that contrary to the susy case there is a crucial change of sign which prevent the cancellation). We complete the discussion by making the complete sum over the spin structures, to get a modular invariant result which contributes to the integrand of two-loop cosmological cosntant, eq. (77).

Thus unfortunately we find that at two loops the same computation showing that the cosmological constant is zero when supersymmetry is unbroken gives a result different from zero in absence of supersymmetry, at least for those models which we have been able to work out explicitely. To be precise, this computation is done for the amplitude before the integration over the moduli of the genus two Riemann surface. However all the proofs appeared so far have been done in this way (including those under question 
for the nonsusy string theories), and indeed there is no indication that the final integration over the moduli should vanish. Thus our conclusion is that the issue of getting realistic and viable models from Superstring is still an unsolved, possibly the main unsolved problem in this context.

\section{A review of the free fermionic construction of type II superstring theories}

In this section we review the free fermionic construction of lower dimensional superstring models [10, 11]. The presentation is tailored to the type II superstring theory.

For type II superstring theory in $D$ dimensions, we have the following list of two-dimensional fields appearing in the construction of the models:

$X^{\mu}, \psi^{\mu}, \tilde{\psi}^{\mu}(\mu=0,1, \cdots, D-1)$ which have a space-time interpretaion;

$\chi^{I}, y^{I}, \omega^{I}, I=1, \cdots, 10-D$ which are $3(10-D)$ free left (holomorphic) Majorana fermions;

$\tilde{\chi}^{I}, \tilde{y}^{I}, \tilde{\omega}^{I}, I=1, \cdots, 10-D$ which are $3(10-D)$ free right (anti-holomorphic) Majorana fermions.

The left supercurrent is realized nonlinearly by these fields as [12]

$$
\begin{aligned}
J & =\psi \cdot \partial X+2 c \partial \beta-\gamma b+3 \partial c \beta+\sum_{I=1}^{10-D} \chi^{I} y^{I} \omega^{I}, \\
& \equiv J_{m}+J_{g h}+J_{\chi},
\end{aligned}
$$

and similarly for the right supercurrent $\tilde{J}$. As it is well-known, fermionic fields have non-trivial boundary conditions. For different boundary conditions these fermionic fields give different contributions to the partition function. The partition function should be modular invariant, guarantee the cancellation of gauge and gravitational anomalies (in the low energy or field theory limit of string theory). How modular invariance restricts the possible choice of boundary conditions was discussed in [10, 11]. By studying the one-loop partition function and its multi-loop counterparts, they found a set of rules of constructing modular invariant string theories. By using their rules on can construct a lot of consistent models. Let's briefly review their construction, setting $D=4$ in what follows. 
To start with let us first recall some notations. For the torus it can be represented by a flat parallelogram in the complex plane with side 1 and $\tau$ corresponding to its two non-contractible loops. We denote the one-loop spin structure as $\left[\begin{array}{l}\alpha \\ \beta\end{array}\right]$ where $\alpha$ and $\beta$ are subsets of $F$ (三 the set of all the fermions) containing those fermions that are periodic around 1 and $\tau$ respectively. For any set $\alpha$ of fermions $(\alpha \subseteq F)$ we define its characteric function as

$$
\alpha(f)=1, \quad \text { if } \quad f \in \alpha ; \quad \alpha(f)=0, \quad \text { otherwise. }
$$

We also define addition and multiplication of fermion sets as ordinary addition and multiplication modulo 2 of their characteristic functions. Thus addition is the symmetric difference and multiplication the intersection:

$$
\begin{aligned}
& \alpha+\alpha^{\prime}=\alpha \cup \alpha^{\prime}-\alpha \cap \alpha^{\prime}, \\
& \alpha \alpha^{\prime}=\alpha \cap \alpha^{\prime} .
\end{aligned}
$$

The one-loop vacuum amplitude can be written as

$$
Z_{\text {one-loop }}=\int \frac{d^{2} \tau}{(\operatorname{Im} \tau)^{\frac{D}{2}+1}|\eta|^{24}} \sum_{\alpha, \beta} C\left[\begin{array}{l}
\alpha \\
\beta
\end{array}\right] \prod_{f \in F^{\prime}} \Theta^{1 / 2}\left[\begin{array}{c}
\alpha(f) \\
\beta(f)
\end{array}\right](\tau) .
$$

Here $\eta(\tau)$ is the Didekin eta function; $\Theta\left[\begin{array}{c}\epsilon \\ \epsilon^{\prime}\end{array}\right]$ is the Jacobi theta function with characteristic $\left[\begin{array}{c}\epsilon / 2 \\ \epsilon^{\prime} / 2\end{array}\right]$. By slight abuse of notation we suppress the fact that this theta function should be complex conjugated when $f$ is a right (anti-holomorphic) fermion. For later convenience, we also define $F^{\prime}$ as the set of all "transverse" fermions, i.e. $F$ minus two $\psi^{\mu}$ 's and two $\tilde{\psi}^{\mu}$ 's. ( For later convenience, we also use $F_{\mathrm{L}}$ and $F_{\mathrm{R}}$ to denote all the left fermions and right fermions respectively. $F_{\mathrm{L}}^{\prime}$ to denote all the left fermions minus two $\psi^{\mu}$ 's and similarly for $F_{\mathrm{R}}^{\prime}$ ).

It is important to note that in the 1-loop case there are no superghost zero modes (in the even spin structures; the odd spin structure gives trivially a vanishing contribution to the cosmological constant). Thus, in the expression for $Z_{\text {one-loop }}$ there is no factor containing the supercurrent (or picture changing operators) correlator. Instead, in the two loops case, since there are 
superghost zero modes also for the even spin structures, a supercurrent correlator will appear inside the sum over the spin structures defining $Z_{\text {two-loops }}$. This correlator will play a crucial role in the issue whether the cosmological constant is also zero at two loops.

Finally the coefficients $C\left[\begin{array}{l}\alpha \\ \beta\end{array}\right]$ takes values equal to either +1 or -1 which should be determined from modular invariance.

By modular invariance, the following conditions on the coefficients $C\left[\begin{array}{l}\alpha \\ \beta\end{array}\right]$ are derived [10, 11]:

$$
\begin{aligned}
& C\left[\begin{array}{l}
\alpha \\
\beta
\end{array}\right]=\varepsilon_{\alpha} C\left[\begin{array}{c}
\alpha \\
\alpha+\beta+F
\end{array}\right], \\
& C\left[\begin{array}{l}
\alpha \\
\beta
\end{array}\right]=\varepsilon_{\alpha \beta}^{2} C\left[\begin{array}{l}
\beta \\
\alpha
\end{array}\right], \\
& C\left[\begin{array}{l}
\alpha_{1} \\
\beta_{1}
\end{array} \cdots \alpha_{g}\right. \\
& C\left[\begin{array}{l}
\alpha \\
\beta
\end{array}\right]=C\left[\begin{array}{l}
\alpha^{\prime} \\
\beta^{\prime}
\end{array}\right]=(-1)^{\alpha(\psi, \tilde{\psi})+\alpha^{\prime}(\psi, \tilde{\psi})} \varepsilon_{\alpha \alpha^{\prime}}^{2} C\left[\begin{array}{c}
\alpha \\
\beta+\alpha^{\prime}
\end{array}\right] C\left[\begin{array}{c}
\alpha^{\prime} \\
\beta^{\prime}+\alpha
\end{array}\right],
\end{aligned}
$$

where $\alpha(\psi, \tilde{\psi})=\alpha(\psi)+\alpha(\tilde{\psi}), \varepsilon_{\alpha}=\exp \left(\frac{i \pi n(\alpha)}{8}\right)$, etc. and $n(\alpha)$ is the difference between the number of left fermions in the set $\alpha$ (not counting the two longitudinal fermions if $\psi^{\mu}$ 's and/or $\tilde{\psi}^{\mu}$ 's are in $\alpha$, see below) and the number of right fermions in $\alpha$. These equations are all the equations required for modular invariance to all loops.

The analysis of these equations is rather lengthly [10], but the results can be presented with reasonable dispatch. The sets of fermions which enter the summation in eq. (9) form an additive group $\Xi$ of subsets of $F$, containing in particular $F$ and the empty set $\emptyset$. The precise choice of $\Xi$ and $C\left[\begin{array}{l}\alpha \\ \beta\end{array}\right]$ 's define the theory. This choice is restricted by a series of consistency conditions, which are derived from eqs. (10)-(13). Firstly, admissible spin structure assignments to the fermions must be such that the spercurrent $J$ (and also for $\tilde{J}$ ) has also a well-defined spin structure. In particular this implies that for any assignment $C\left[\begin{array}{l}\alpha \\ \beta\end{array}\right]$ the supercurrent $J$ and the superghosts, as well 
as the $D$ fermion field $\psi^{\mu}$ 's all have the same spin structure $\left[\begin{array}{l}\alpha\left(\psi^{\mu}\right) \\ \beta\left(\psi^{\mu}\right)\end{array}\right]$. All the $D$ fermion field $\psi^{\mu}$ 's are either all in the set $\alpha$ or all not in the set. The second condition is that for all $\alpha, \alpha^{\prime}$, etc. $\in \Xi$ :

$$
\begin{aligned}
& n(\alpha)=0 \bmod 8, \\
& n\left(\alpha \alpha^{\prime}\right)=0 \bmod 4, \\
& n\left(\alpha \alpha^{\prime} \alpha^{\prime \prime} \alpha^{\prime \prime \prime}\right)=0 \bmod 2 .
\end{aligned}
$$

Finally the coefficients $C\left[\begin{array}{l}\alpha \\ \beta\end{array}\right]$ must obey

$$
\begin{aligned}
C\left[\begin{array}{l}
\alpha \\
\beta
\end{array}\right] & =\epsilon_{\alpha \beta}^{2} C\left[\begin{array}{l}
\beta \\
\alpha
\end{array}\right], \\
C\left[\begin{array}{l}
\alpha \\
\alpha
\end{array}\right] & =\epsilon_{\alpha} C\left[\begin{array}{l}
\alpha \\
F
\end{array}\right], \\
C\left[\begin{array}{c}
\alpha \\
\beta+\gamma
\end{array}\right] & =(-1)^{\alpha(\psi, \tilde{\psi})} C\left[\begin{array}{l}
\alpha \\
\beta
\end{array}\right] C\left[\begin{array}{l}
\alpha \\
\gamma
\end{array}\right] .
\end{aligned}
$$

Note that conditions (14) ensure the consistency of eq. (15). For our later use one can derive the following useful formula:

$$
C\left[\begin{array}{c}
\alpha+\beta \\
\gamma
\end{array}\right]=(-1)^{\gamma\left(\psi^{\mu}, \tilde{\psi}^{\mu}\right)} \exp \left\{\frac{i \pi}{2} n(\alpha \beta \gamma)\right\} C\left[\begin{array}{l}
\alpha \\
\gamma
\end{array}\right] C\left[\begin{array}{l}
\beta \\
\gamma
\end{array}\right]
$$

By carefully analysing the spectrum of non-interacting string states, it was found that a string model has a massless gravitino if and only if there exists a set $S \in \Xi$ such that the following two conditions are satisfied [10]:

1. $S$ is a set of precisely eight left fermions including $\psi^{\mu}$ (not counting the two longitudinal fermions) or a set of precisely eight right fermions including $\tilde{\psi}^{\mu}$

2. for any set $X \in \Xi$ satisfying $X \cap S=\emptyset$, we must have

$$
C\left[\begin{array}{l}
S \\
X
\end{array}\right]=-(-1)^{X\left(\psi^{\mu}, \tilde{\psi}^{\mu}\right)} .
$$

The model we will study in detail in this paper is constructed in [6] and it is given by the following generating sets:

$$
b_{0}=F=\left\{\psi^{\mu=0, \cdots, 3}, \chi^{I=1, \cdots, 6}, y^{I=1, \cdots, 6}, \omega^{I=1, \cdots, 6},\right.
$$




$$
\begin{aligned}
& \left.\tilde{\psi}^{\mu=0, \cdots, 3}, \tilde{\chi}^{I=1, \cdots, 6}, \tilde{y}^{I=1, \cdots, 6}, \tilde{\omega}^{I=1, \cdots, 6}\right\}, \\
b_{1}= & \left\{\psi^{\mu=0, \cdots, 3}, \chi^{I=1, \cdots, 6}\right\}, \\
b_{2}= & \left\{\tilde{\psi}^{\mu=0, \cdots, 3}, \tilde{\chi}^{I=1, \cdots, 6}\right\}, \\
b_{3}= & \left\{\chi^{I=1, \cdots, 4}, y^{I=1, \cdots, 4}, \tilde{y}^{I=1, \cdots, 4}, \tilde{\omega}^{I=1, \cdots, 4}\right\}, \\
b_{4}= & \left\{y^{I=1, \cdots, 4}, \omega^{I=1, \cdots, 4}, \tilde{\chi}^{I=1, \cdots, 4}, \tilde{y}^{I=1, \cdots, 4}\right\} .
\end{aligned}
$$

To completely fix the model we must specify what all these coefficients

$C\left[\begin{array}{l}\alpha \\ \beta\end{array}\right]$ 's are. The strategy is the following: all models obtained by omitting one or two generating sets $b_{3}$ and/or $b_{4}$ are supersymmetric. In particular the model generated by the sets $b_{0,1,2}$ has $(4,4)$ supersymmetry. This requires:

$$
C\left[\begin{array}{l}
b_{1} \\
b_{2}
\end{array}\right]=C\left[\begin{array}{l}
b_{2} \\
b_{1}
\end{array}\right]=1
$$

(See Appendix A.) Notice also that

$$
b_{1} \cap b_{4}=\emptyset, \quad \text { and } \quad b_{2} \cap b_{3}=\emptyset,
$$

from the general analysis, we will have a non-supersymmetric model if we have

$$
C\left[\begin{array}{l}
b_{1} \\
b_{4}
\end{array}\right]=1, \quad \text { and } \quad C\left[\begin{array}{l}
b_{2} \\
b_{3}
\end{array}\right]=1
$$

We will not analyse the spectrum of this model [6] here. An analysis of the gravitini is given in Appendix A. For this model it was proved that the one-loop cosmological constant is zero even before the integration over the moduli space parameter $\tau$. We will give a complete proof in the next section. The method will be used in our explicit computation of the two-loop cosmological constant (which is non-vanishing before the integration over the moduli space).

\section{One-loop cosmological constant}

The one-loop cosmological constant was given in (9). What we will prove is that the cosmological constant is zero even before the integration over the 
moduli $\tau$. So we can first consider the following expression:

$$
\begin{aligned}
V= & \sum_{\alpha, \beta \in \Xi} C\left[\begin{array}{l}
\alpha \\
\beta
\end{array}\right] \prod_{f \in F^{\prime}} \Theta^{1 / 2}\left[\begin{array}{l}
\alpha(f) \\
\beta(f)
\end{array}\right](\tau) \\
= & \sum_{\alpha, \beta \in \Xi_{0}} \sum_{n, m, \tilde{n}, \tilde{m}=0}^{1} C\left[\begin{array}{l}
\alpha+n b_{3}+m b_{4} \\
\beta+\tilde{n} b_{3}+\tilde{m} b_{4}
\end{array}\right] \\
& \times \prod_{f \in F^{\prime}} \Theta^{1 / 2}\left[\begin{array}{l}
\left(\alpha+n b_{3}+m b_{4}\right)(f) \\
\left(\beta+\tilde{n} b_{3}+\tilde{m} b_{4}\right)(f)
\end{array}\right]^{1 / 2}(\tau) .
\end{aligned}
$$

In the above expression we have separated the summation over $b_{3}$ and $b_{4}$ from the summation over $\Xi_{0}$ which is defined as the set generated by the sets $b_{0,1,2}: \Xi_{0}=\left\{\sum_{i=0}^{2} n_{i} b_{i} \mid n_{i}=0,1\right\}$. Now setting $\alpha \rightarrow \alpha+n_{1} b_{1}+m_{1} b_{2}$ and $\beta \rightarrow \beta+n_{2} b_{1}+m_{2} b_{2}$, we do a resummation over $b_{1}$ and $b_{2}$ :

$$
\begin{aligned}
V= & \frac{1}{2^{4}} \sum_{\alpha, \beta \in \Xi_{0}} \sum_{n, m, \tilde{n}, \tilde{m}=0}^{1} \sum_{n_{1}, n_{2}, m_{1}, m_{2}=0}^{1} C\left[\begin{array}{c}
\alpha+n b_{3}+m b_{4}+n_{1} b_{1}+m_{1} b_{2} \\
\beta+\tilde{n} b_{3}+\tilde{m} b_{4}+n_{2} b_{1}+m_{2} b_{2}
\end{array}\right] \\
& \times \prod_{f \in F^{\prime}} \Theta^{1 / 2}\left[\begin{array}{c}
\left(\alpha+n b_{3}+m b_{4}+n_{1} b_{1}+m_{1} b_{2}\right)(f) \\
\left(\beta+\tilde{n} b_{3}+\tilde{m} b_{4}+n_{2} b_{1}+m_{2} b_{2}\right)(f)
\end{array}\right](\tau) \\
\equiv & \frac{1}{2^{4}} \sum_{\alpha, \beta \in \Xi_{0}} \sum_{n, m, \tilde{n}, \tilde{m}=0}^{1} V\left[\begin{array}{l}
\alpha+n b_{3}+m b_{4} \\
\beta+\tilde{n} b_{3}+\tilde{m} b_{4}
\end{array}\right]
\end{aligned}
$$

defining $V[]$ in an obvious notation. It is easy to prove the following property for $V[]$ :

$$
V\left[\begin{array}{c}
\alpha+n b_{3}+m b_{4} \\
\beta+\tilde{n} b_{3}+\tilde{m} b_{4}
\end{array}\right]=V\left[\begin{array}{c}
\alpha+n b_{3}+m b_{4}+n_{1} b_{1}+m_{1} b_{2} \\
\beta+\tilde{n} b_{3}+\tilde{m} b_{4}+n_{2} b_{1}+m_{2} b_{2}
\end{array}\right]
$$

for any $n_{1}, n_{2}, m_{1}, m_{2}=0,1$ and $\alpha, \beta \in \Xi_{0}$. Now we define a subset $\Xi_{0}^{\prime}$ of $\Xi_{0}: \Xi_{0}^{\prime} \equiv\left\{\alpha \in \Xi_{0} \mid \alpha \cap b_{1}=\alpha \cap b_{2}=\emptyset\right\}=\left\{\emptyset, F+b_{1}+b_{2}\right\}$. By using the the above property of $V$ we can restrict the summation over $\alpha$ and $\beta$ to $\Xi_{0}^{\prime}$ :

$$
V=\sum_{\alpha, \beta \in \Xi_{0}^{\prime}} \sum_{n, m, \tilde{n}, \tilde{m}=0,1} V\left[\begin{array}{c}
\alpha+n b_{3}+m b_{4} \\
\beta+\tilde{n} b_{3}+\tilde{m} b_{4}
\end{array}\right] .
$$

In the following we will prove that all these $V$ []'s are identically zero. Before doing this let us first classify all these different $V$ []'s according to their 
modular transformation property. In fact all these $V$ []'s can be classified into five categories. They are:

1) $V\left[\begin{array}{l}\alpha \\ \beta\end{array}\right]$

2) $V\left[\begin{array}{c}\alpha+b_{3} \\ \beta\end{array}\right], V\left[\begin{array}{c}\alpha \\ \beta+b_{3}\end{array}\right]$ and $V\left[\begin{array}{c}\alpha+b_{3} \\ \beta+b_{3}\end{array}\right]$;

3-4) obtained from the above by substitution $b_{3} \rightarrow b_{4}$ or $b_{3} \rightarrow b_{3}+b_{4}$;

5) $V\left[\begin{array}{c}\alpha+b_{3} \\ \beta+b_{4}\end{array}\right], V\left[\begin{array}{c}\alpha+b_{3} \\ \beta+b_{3}+b_{4}\end{array}\right], V\left[\begin{array}{c}\alpha+b_{4} \\ \beta+b_{3}+b_{4}\end{array}\right], V\left[\begin{array}{c}\alpha+b_{4} \\ \beta+b_{3}\end{array}\right]$, $V\left[\begin{array}{c}\alpha+b_{3}+b_{4} \\ \beta+b_{3}\end{array}\right], V\left[\begin{array}{c}\alpha+b_{3}+b_{4} \\ \beta+b_{4}\end{array}\right]$.

All the $V[$ ]'s in the same category can be transformed into each other by modular transformations. This can easily be shown by making use of the following generating modular transformations at one-loop:

$$
\begin{aligned}
& \Theta\left[\begin{array}{l}
a \\
b
\end{array}\right](\tau) \stackrel{\mathrm{S}}{\longrightarrow} \Theta\left[\begin{array}{l}
b \\
a
\end{array}\right](-1 / \tau), \\
& \Theta\left[\begin{array}{l}
a \\
b
\end{array}\right](\tau) \stackrel{\mathrm{T}}{\longrightarrow} \Theta\left[\begin{array}{c}
a \\
a+b+1
\end{array}\right](\tau+1) .
\end{aligned}
$$

The property of the coefficient $C[$ ]'s ensures that all $V$ []'s transform nicely and we have

$$
\begin{aligned}
& V\left[\begin{array}{l}
\alpha \\
\beta
\end{array}\right](\tau) \stackrel{\mathrm{S}}{\longrightarrow} V\left[\begin{array}{l}
\beta \\
\alpha
\end{array}\right](-1 / \tau), \\
& V\left[\begin{array}{l}
\alpha \\
\beta
\end{array}\right](\tau) \stackrel{\mathrm{T}}{\longrightarrow} V\left[\begin{array}{c}
\alpha \\
\alpha+\beta+b_{0}
\end{array}\right](\tau+1) .
\end{aligned}
$$

The following demonstrates how we transform $V\left[\begin{array}{c}\alpha+b_{3} \\ \beta+b_{4}\end{array}\right]$ to $V\left[\begin{array}{c}\tilde{\alpha}+b_{3}+b_{4} \\ \tilde{\beta}+b_{4}\end{array}\right]$ by a series of modular transformations:

$$
\begin{aligned}
& V\left[\begin{array}{l}
\alpha+b_{3} \\
\beta+b_{4}
\end{array}\right](\tau) \stackrel{\mathrm{S}}{\longrightarrow} V\left[\begin{array}{l}
\beta+b_{4} \\
\alpha+b_{3}
\end{array}\right](-1 / \tau) \\
& \stackrel{\mathrm{T}}{\longrightarrow} V\left[\begin{array}{c}
\beta+b_{4} \\
\alpha+\beta+b_{0}+b_{3}+b_{4}
\end{array}\right](-1 / \tau+1)
\end{aligned}
$$




$$
\begin{aligned}
& \stackrel{\mathrm{S}}{\longrightarrow} V\left[\begin{array}{c}
\alpha+\beta+b_{0}+b_{3}+b_{4} \\
\beta+b_{4}
\end{array}\right](\tau /(1-\tau)) \\
& =V\left[\begin{array}{c}
\alpha+\beta+b_{0}+b_{1}+b_{2}+b_{3}+b_{4} \\
\beta+b_{4}
\end{array}\right](\tau /(1-\tau)) \\
& \equiv V\left[\begin{array}{c}
\tilde{\alpha}+b_{3}+b_{4} \\
\tilde{\beta}+b_{4}
\end{array}\right](\tau /(1-\tau))
\end{aligned}
$$

with $\tilde{\alpha}=\alpha+\beta+b_{0}+b_{1}+b_{2}$ and $\tilde{\beta}=\beta$. One easily ckecks that

$$
\alpha, \beta \in \Xi_{0}^{\prime} \Longrightarrow \tilde{\alpha} \in \Xi_{0}^{\prime}
$$

After this detour we can prove the vanishing of all $V$ []'s in one category by just proving the vanishing of just one of the $V[]$ in this category. Modular transformation ensures the vanishing of all the other $V$ []'s because they are related with each other by modular transormations.

The vanishing of $V$ []'s in the first 4 categories is automatic because of supersymmetry. On the other hand the vanishing of $V[]$ 's in the fifth categories is because there always exists an odd spin structure in the theta function product. Let us prove these assertions in the following.

To prove $V\left[\begin{array}{l}\alpha \\ \beta\end{array}\right]=0$, let us first recall the definition of it given in (23):

$$
V\left[\begin{array}{l}
\alpha \\
\beta
\end{array}\right]=\sum_{n_{i}, m_{i}=0}^{1} C\left[\begin{array}{c}
\alpha+n_{1} b_{1}+m_{1} b_{2} \\
\beta+n_{2} b_{1}+m_{2} b_{2}
\end{array}\right] \prod_{f \in F^{\prime}} \Theta^{1 / 2}\left[\begin{array}{c}
\left(\alpha+n_{1} b_{1}+m_{1} b_{2}\right)(f) \\
\left(\beta+n_{2} b_{1}+m_{2} b_{2}\right)(f)
\end{array}\right] .
$$

Now we separate the transverse fermions $F^{\prime}$ into three non-intersecting subsets $b_{1}, b_{2}$ and $F^{\prime}+b_{1}+b_{2}$. Then we have ${ }^{1}$

$$
\begin{aligned}
V\left[\begin{array}{l}
\alpha \\
\beta
\end{array}\right]= & \sum_{n_{i}, m_{i}=0}^{1} C\left[\begin{array}{l}
\alpha+n_{1} b_{1}+m_{1} b_{2} \\
\beta+n_{2} b_{1}+m_{2} b_{2}
\end{array}\right] \prod_{f \in b_{1}} \Theta^{1 / 2}\left[\begin{array}{l}
n_{1} \\
n_{2}
\end{array}\right](\tau) \\
& \times \prod_{f \in b_{2}} \bar{\Theta}^{1 / 2}\left[\begin{array}{l}
m_{1} \\
m_{2}
\end{array}\right](\bar{\tau}) \prod_{f \in F^{\prime}+b_{1}+b_{2}} \Theta^{1 / 2}\left[\begin{array}{c}
\alpha(f) \\
\beta(f)
\end{array}\right] \\
= & \sum_{n_{i}, m_{i}=0}^{1} C\left[\begin{array}{l}
\alpha+n_{1} b_{1}+m_{1} b_{2} \\
\beta+n_{2} b_{1}+m_{2} b_{2}
\end{array}\right] \Theta^{4}\left[\begin{array}{c}
n_{1} \\
n_{2}
\end{array}\right](\tau) \bar{\Theta}^{4}\left[\begin{array}{l}
m_{1} \\
m_{2}
\end{array}\right](\bar{\tau})
\end{aligned}
$$

\footnotetext{
${ }^{1}$ Remember $\alpha \cap b_{i}=\beta \cap b_{i}=\emptyset, i=1,2$.
} 


$$
\times \prod_{f \in F^{\prime}+b_{1}+b_{2}} \Theta^{1 / 2}\left[\begin{array}{c}
\alpha(f) \\
\beta(f)
\end{array}\right] .
$$

Noticing $\alpha \cap b_{i}=\beta \cap b_{i}=\emptyset$, one can derive the following:

$$
\begin{aligned}
& C\left[\begin{array}{c}
\alpha \\
n b_{1}
\end{array}\right]=C\left[\begin{array}{c}
\alpha \\
n b_{2}
\end{array}\right]=(-1)^{n}, \\
& C\left[\begin{array}{c}
\alpha+n_{1} b_{1}+m_{1} b_{2} \\
\beta+n_{2} b_{1}+m_{2} b_{2}
\end{array}\right]=(-1)^{n_{1}+n_{2}+m_{1}+m_{2}} C\left[\begin{array}{c}
\alpha \\
\beta
\end{array}\right] .
\end{aligned}
$$

By using these results in (33) we have

$$
\begin{aligned}
V\left[\begin{array}{l}
\alpha \\
\beta
\end{array}\right] & \propto \sum_{n_{i}=0}^{1}(-1)^{n_{1}+n_{2}} \Theta^{4}\left[\begin{array}{l}
n_{1} \\
n_{2}
\end{array}\right](\tau) \sum_{m_{i}=0}^{1}(-1)^{m_{1}+m_{2}} \Theta^{4}\left[\begin{array}{l}
m_{1} \\
m_{2}
\end{array}\right](\bar{\tau}) \\
& =\left(\Theta^{4}\left[\begin{array}{l}
0 \\
0
\end{array}\right](\tau)-\Theta^{4}\left[\begin{array}{l}
0 \\
1
\end{array}\right](\tau)-\Theta^{4}\left[\begin{array}{l}
1 \\
0
\end{array}\right](\tau)+\Theta^{4}\left[\begin{array}{l}
1 \\
1
\end{array}\right](\tau)\right) \times(C . C .) \\
& =0
\end{aligned}
$$

by making use of the well-known identity:

$$
\Theta^{4}\left[\begin{array}{l}
0 \\
0
\end{array}\right](\tau)-\Theta^{4}\left[\begin{array}{l}
0 \\
1
\end{array}\right](\tau)-\Theta^{4}\left[\begin{array}{l}
1 \\
0
\end{array}\right](\tau)=0
$$

and $\Theta\left[\begin{array}{l}1 \\ 1\end{array}\right](\tau)=0$. In eq. (36),$(C . C$.$) denotes complex conjugation.$

To prove $V\left[\begin{array}{c}\alpha+b_{3} \\ \beta\end{array}\right]=0$, one derives

$$
C\left[\begin{array}{c}
\alpha+b_{3}+n_{1} b_{1}+m_{1} b_{2} \\
\beta+n_{2} b_{1}+m_{2} b_{2}
\end{array}\right]=(-1)^{n_{1}+m_{1}} C\left[\begin{array}{c}
\alpha+b_{3} \\
\beta
\end{array}\right] C\left[\begin{array}{c}
b_{3} \\
n_{2} b_{1}
\end{array}\right] C\left[\begin{array}{c}
b_{3} \\
m_{2} b_{2}
\end{array}\right]
$$

and we have

$$
\begin{aligned}
V\left[\begin{array}{c}
\alpha+b_{3} \\
\beta
\end{array}\right] \propto & \sum_{n_{i}, m_{i}=0}^{1}(-1)^{n_{1}+m_{1}} C\left[\begin{array}{c}
b_{3} \\
n_{2} b_{1}
\end{array}\right] C\left[\begin{array}{c}
b_{3} \\
m_{2} b_{2}
\end{array}\right] \Theta^{2}\left[\begin{array}{l}
n_{1} \\
n_{2}
\end{array}\right](\tau) \\
& \times \Theta^{2}\left[\begin{array}{c}
n_{1}+1 \\
n_{2}
\end{array}\right](\tau) \Theta^{4}\left[\begin{array}{c}
m_{1} \\
m_{2}
\end{array}\right](\bar{\tau})
\end{aligned}
$$




$$
\begin{aligned}
& \propto \sum_{n_{1}=0}^{1}(-1)^{n_{1}} \Theta^{2}\left[\begin{array}{c}
n_{1} \\
0
\end{array}\right](\tau) \Theta^{2}\left[\begin{array}{c}
n_{1}+1 \\
0
\end{array}\right](\tau) \\
& =\Theta^{2}\left[\begin{array}{l}
0 \\
0
\end{array}\right](\tau) \Theta^{2}\left[\begin{array}{l}
1 \\
0
\end{array}\right](\tau)-\Theta^{2}\left[\begin{array}{l}
1 \\
0
\end{array}\right](\tau) \Theta^{2}\left[\begin{array}{l}
0 \\
0
\end{array}\right](\tau) \\
& =0
\end{aligned}
$$

Here we have used the fact that because $\Theta\left[\begin{array}{l}1 \\ 1\end{array}\right](\tau)=0$, only $n_{2}=0$ might give a possibly non-vanishing contribution to $V$.

Finally the proof of $V\left[\begin{array}{l}\alpha+b_{3} \\ \beta+b_{4}\end{array}\right]=0$ is based on the following results $\left(b \equiv F+b_{1}+b_{2}\right)$ :

$$
\begin{aligned}
\alpha=\beta=\emptyset, & & b_{3} \cap b_{4}=\left\{y^{I=1, \cdots, 4}, \tilde{y}^{I=1, \cdots, 4}\right\}, \\
\alpha=\emptyset, \beta=b, & & b_{3} \cap\left(\beta+b_{4}\right)=\left\{\tilde{\omega}^{I=1, \cdots, 4}\right\} \\
\beta=\emptyset, \alpha=b, & & \left(\alpha+b_{3}\right) \cap b_{4}=\left\{\omega^{I=1, \cdots, 4}\right\} \\
\alpha=\beta=b, & & \left(\alpha+b_{3}\right) \cap\left(\beta+b_{4}\right)=\left\{y^{I}, \tilde{y}^{I}, \omega^{I}, \tilde{\omega}^{I} \mid I=5,6\right\} .
\end{aligned}
$$

By using these results we have

$$
\begin{aligned}
V\left[\begin{array}{c}
\alpha+b_{3} \\
\beta+b_{4}
\end{array}\right] & \propto \prod_{f \in F^{\prime}+b_{1}+b_{2}} \Theta^{1 / 2}\left[\begin{array}{c}
\left(\alpha+b_{3}\right)(f) \\
\left(\beta+b_{4}\right)(f)
\end{array}\right] \\
& \propto \Theta^{2}\left[\begin{array}{l}
1 \\
1
\end{array}\right](\tau) \text { or } \bar{\Theta}^{2}\left[\begin{array}{l}
1 \\
1
\end{array}\right](\bar{\tau})=0
\end{aligned}
$$

In the next section we extend the above reasoning to two-loop computations, but we will find a non-vanishing result for the cosmological constant before the integration over the moduli space.

\section{Two-loop cosmological constant}

As we recalled above, in two-loop computation another factor must be inserted into the sum over the even spin structures, representing the supercurrent correlator related to the occurrence of superghost zero modes (the odd spin structures give a trivially zero contribution in this case too). Its exact 
form is quite involved, however for checking the vanishing of the cosmological constant we only need few relevant facts, which we will recall here. The computation of the two-loop cosmological constant (the left part) follows the standard strategy [3, 13, 8] of first integrating over the supermoduli by choosing the super-Beltrami differentials to be $\delta$-functions in moduli independent points $x_{1,2}$ on the Riemann surface. One thus obtains for the left part of the integrand (to be integrated over the moduli of the surface, represented by three of the branch points $a_{i}$, the other three being fixed by the $S L(2, C)$ projective invariance, see below):

$$
V_{L}=\left(\operatorname{det} \bar{\partial}_{1}\right)^{-D / 2} \operatorname{det} \bar{\partial}_{2}\left(\operatorname{det} \bar{\partial}_{3 / 2}\right)_{s(\beta, \gamma)}^{-1} \frac{\left\langle J\left(x_{1}\right) J\left(x_{2}\right)\right\rangle_{s(\psi)}}{\operatorname{det} \Phi_{a}^{3 / 2}\left(x_{b}\right)} \prod_{f \in F_{\mathrm{L}}}\left(\operatorname{det} \bar{\partial}_{1 / 2}\right)_{s(f)}^{1 / 2} \text {, }
$$

where $\operatorname{det} \bar{\partial}_{j}$ stands for the usual chiral determinant and $\Phi_{a}^{3 / 2}(x), a=1,2$ are the holomorphic 3/2-differentials. All the determinants, the correlators and 3/2-differentials appearing in eq. (45) can be explicitly expressed in the hyperelliptic description, where the genus $g=2$ Riemann surface is represented as lower and upper (compactified) sheets of the function

$$
y^{2}(z)=\prod_{i=1}^{6}\left(z-a_{i}\right)
$$

and the branch points $a_{i}$ define three branch cuts in each sheet. Three $a_{i}$ can be fixed by $S L(2, C)$ projective invariance and the other three are taken to be moduli. The two independent holomorphic one-forms $w_{i}(z)$ can be taken to be

$$
w_{1}(z)=\frac{1}{y(z)}, \quad w_{2}(z)=\frac{z}{y(z)} .
$$

As in ref. [3] we choose the positions $x_{1,2}$ of the super-Beltrami differentials to be the two simple zeros of $w_{1}$, i.e. $x_{1}=\infty$ in the upper sheet and $x_{2}=\infty$ in the lower sheet respectively. In this language the even spin structures are equivalent to ten different splitting of the six branch points $\left\{a_{i}\right\}, i=1, \cdots, 6$ into two non-ordered sets $\left\{A_{i}\right\},\left\{B_{i}\right\}, i=1,2,3$.

With a convenient normalization [3] we have $\operatorname{det} \Phi_{a}^{3 / 2}\left(x_{b}\right)=1$, and the spin-structure dependent part of $\left\langle J\left(x_{1}\right) J\left(x_{2}\right)\right\rangle$ is carried by the expressions

$$
Y_{1}[s(\psi)] \equiv 2\left\langle\psi\left(x_{1}\right) \psi\left(x_{2}\right)\right\rangle_{s(\psi)}=\frac{1}{2} \sum_{i=1}^{3}\left(A_{i}-B_{i}\right)
$$




$$
Y_{2}[s(\psi)] \equiv 4\left\langle\partial \psi\left(x_{1}\right) \psi\left(x_{2}\right)\right\rangle_{s(\psi)}=\frac{1}{2} \sum_{i=1}^{3}\left(A_{i}^{2}-B_{i}^{2}\right),
$$

coming from the $J_{\psi}=\psi \cdot \partial X$ and $J_{\text {gh }}$ part of eq. (5) (see ref. [3] for details). Similarly, the compactified sector contribution is

$$
\left\langle J_{\chi}\left(x_{1}\right) J_{\chi}\left(x_{2}\right)\right\rangle_{s(\psi)}=-\sum_{I=1}^{6} Y_{1}\left[s\left(\chi^{I}\right)\right] Y_{1}\left[s\left(y^{I}\right)\right] Y_{1}\left[s\left(\omega^{I}\right)\right]
$$

With all these expressions in hand we can write

$$
\left\langle J\left(x_{1}\right) J\left(x_{2}\right)\right\rangle_{s(\psi)}=K_{1} Y_{1}[s(\psi)]+K_{2} Y_{2}[s(\psi)]-\sum_{I=1}^{6} Y_{1}\left[s\left(\chi^{I}\right)\right] Y_{1}\left[s\left(y^{I}\right)\right] Y_{1}\left[s\left(\omega^{I}\right)\right] \text {, }
$$

where $K_{i}$ 's are spin structure independent (to be precise $K_{i}$ 's are symmetric for every $a_{i} \leftrightarrow a_{j}$ interchange).

By using the above hyperelliptic representation of the genus 2 Riemann surface, one can compute all the determinants in (45) explicityly and we have the following result for $V_{L}$ :

$$
V_{L}=\frac{1}{(\operatorname{det} K)^{D / 2} \prod_{i<j} a_{i j}}\langle J(r+) J(r-)\rangle_{s(\psi)} \prod_{f \in F_{L}^{\prime}} Q_{s(f)}^{1 / 8},
$$

where we have put $x_{1}=r+$ and $x_{2}=r-$, meaning two arbitrary points (not necessarily at $\infty$ ) on the lower and upper sheets of the complex plane. Also the quantity $Q_{s}$ is proportional (with a spin structure independent factor), by means of the Thomae formula [14] to $\theta_{s}^{4}(0)$. In terms of the above sets $\left\{A_{i}\right\},\left\{B_{i}\right\}$ we have:

$$
Q_{s}=\prod_{i<j}^{3}\left(A_{i}-A_{j}\right)\left(B_{i}-B_{j}\right)
$$

By using this result we can write down the two-loop cosmological constant as follows:

$$
\Lambda=\int \frac{d^{2} \mu}{T^{D / 2} \prod_{i<j}\left|a_{i j}\right|^{2}} \sum_{\alpha, \beta \in \Xi} C\left[\begin{array}{c}
\alpha \\
\beta
\end{array}\right]\langle J(r+) J(r-) \tilde{J}(\bar{s}+) \tilde{J}(\bar{s}-)\rangle_{s(\psi, \tilde{\psi})} \prod_{f \in F^{\prime}} Q_{s(f)}^{1 / 8},
$$


where $d^{2} \mu=\frac{\prod_{i=1}^{6} d^{2} a_{i}}{\prod_{i<j}\left|a_{i j}\right|^{2} d V}$ is the measure of the two-loop moduli space and $d V=\frac{d^{2} a_{i} d^{2} a_{j} d^{2} a_{k}}{\left|a_{i j} a_{j k} a_{k i}\right|^{2}}$ is the infinitesimal volume of the $S L(2, C)$ tranformation.

In type II superstring the correlator $\langle J J \tilde{J} \tilde{J}\rangle$ is not completely left-right factorized. However as far as its spin structure dependence is concerned (i.e. what is relevant to check the zero of the cosmological constant) it is a sum of factorized pieces. Therefore, since we will check separately the terms containing $Y_{1}$ and $Y_{2}$, we can assume the supercurrent correlator, denoted for short $X_{\text {long }, 3 / 2}$, to be effectively factorized: $X_{\text {long }, 3 / 2} \equiv\langle J J \tilde{J} \tilde{J}\rangle=\langle J J\rangle\langle\tilde{J} \tilde{J}\rangle$. Only when we want the complete expression for the cosmological constant, we should take into account this left-right coupling.

\section{Organization of the computation}

At two loops one can do a resummation over $b_{1}$ and $b_{2}$ just as we did at one loop order, by making use of the same subsets $\Xi_{0}$ and $\Xi_{0}^{\prime}$ introduced at one loop. We have

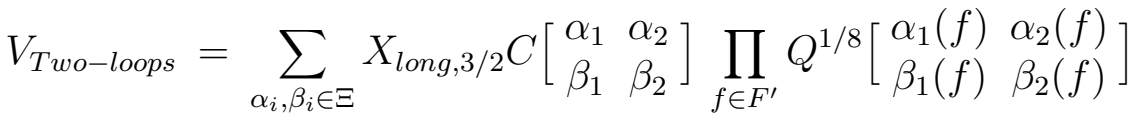

$$
\begin{aligned}
& =\sum_{\alpha_{i}, \beta_{i} \in \Xi_{0}} \sum_{n_{i}, m_{i}, \tilde{n}_{i}, \tilde{m}_{i}=0}^{1} X_{l o n g, 3 / 2} C\left[\begin{array}{cc}
\alpha_{1}+n_{1} b_{3}+m_{1} b_{4} & \alpha_{2}+n_{2} b_{3}+m_{2} b_{4} \\
\beta_{1}+\tilde{n}_{1} b_{3}+\tilde{m}_{1} b_{4} & \beta_{2}+\tilde{n}_{2} b_{3}+\tilde{m}_{2} b_{4}
\end{array}\right] \\
& \times \prod_{f \in F^{\prime}} Q^{1 / 8}\left[\begin{array}{ll}
\left(\alpha_{1}+n_{1} b_{3}+m_{1} b_{4}\right)(f) & \left(\alpha_{2}+n_{2} b_{3}+m_{2} b_{4}\right)(f) \\
\left(\beta_{1}+\tilde{n}_{1} b_{3}+\tilde{m}_{1} b_{4}\right)(f) & \left(\beta_{2}+\tilde{n}_{2} b_{3}+\tilde{m}_{2} b_{4}\right)(f)
\end{array}\right] \\
& =\sum_{\alpha_{i}, \beta_{i} \in \Xi_{0}^{\prime}} \sum_{n_{i}, m_{i}, \tilde{n}_{i}, \tilde{m}_{i}=0}^{1} V\left[\begin{array}{cc}
\alpha_{1}+n_{1} b_{3}+m_{1} b_{4} & \alpha_{2}+n_{2} b_{3}+m_{2} b_{4} \\
\beta_{1}+\tilde{n}_{1} b_{3}+\tilde{m}_{1} b_{4} & \beta_{2}+\tilde{n}_{2} b_{3}+\tilde{m}_{2} b_{4}
\end{array}\right],
\end{aligned}
$$

where

$$
\begin{gathered}
V\left[\begin{array}{cc}
\alpha_{1} & \alpha_{2} \\
\beta_{1} & \beta_{2}
\end{array}\right] \equiv \sum_{n_{i}, m_{i}, \tilde{n}_{i}, \tilde{m}_{i}=0}^{1} X_{\text {long }, 3 / 2} C\left[\begin{array}{cc}
\alpha_{1}+n_{1} b_{1}+m_{1} b_{2} & \alpha_{2}+n_{2} b_{1}+m_{2} b_{2} \\
\beta_{1}+\tilde{n}_{1} b_{1}+\tilde{m}_{1} b_{2} & \beta_{2}+\tilde{n}_{2} b_{1}+\tilde{m}_{2} b_{2}
\end{array}\right] \\
\times \prod_{f \in F^{\prime}} Q^{1 / 8}\left[\begin{array}{cc}
\left(\alpha_{1}+n_{1} b_{1}+m_{1} b_{2}\right)(f) & \left(\alpha_{2}+n_{2} b_{1}+m_{2} b_{2}\right)(f) \\
\left(\beta_{1}+\tilde{n}_{1} b_{1}+\tilde{m}_{1} b_{2}\right)(f) & \left(\beta_{2}+\tilde{n}_{2} b_{1}+\tilde{m}_{2} b_{2}\right)(f)
\end{array}\right] .
\end{gathered}
$$

As in one-loop computation we classify all the $V$ []'s into 6 categories (see Appendix B). They are $\left(\alpha_{i}, \beta_{i} \in \Xi_{0}^{\prime}\right)$ : 
1) $V\left[\begin{array}{cc}\alpha_{1} & \alpha_{2} \\ \beta_{1} & \beta_{2}\end{array}\right]$

2) $V\left[\begin{array}{cc}\alpha_{1}+b_{3} & \alpha_{2} \\ \beta_{1} & \beta_{2}\end{array}\right]$ and 14 other $V$ []'s obtained by two-loop modular transformations;

3-4) obtained from the above by substitution $b_{3} \rightarrow b_{4}$ or $b_{3} \rightarrow b_{3}+b_{4}$;

5) $V\left[\begin{array}{cc}\alpha_{1}+b_{3} & \alpha_{2} \\ \beta_{1}+b_{4} & \beta_{2}\end{array}\right]$ and 119 other $V$ []'s obtained by two-loop modular transformations;

6) $V\left[\begin{array}{cc}\alpha_{1}+b_{3} & \alpha_{2}+b_{4} \\ \beta_{1} & \beta_{2}\end{array}\right]$ and 89 other $V$ []'s obtained by two-loop modular transformations.

The total number of different $V$ []'s (not counting different $\alpha_{i}, \beta_{j} \in \Xi_{0}^{\prime}$ ) is $\left(2^{2}\right)^{4}=256$ which is the right number of different $V$ []'s in the above classification.

\section{Computing the various terms}

\subsection{The susy preserving terms.}

The $V$ []'s in the first 4 categories are zero because of supersymmetry. This was proved rigorously in [4]. We will give the proof in the Appendix C. This is a useful exercise because we will see quite clearly the difference between the supersymmetric model and the non-supersymmetric model.

We note that this proof makes use of the following identities $(i=1,2)$ :

$$
\begin{aligned}
& \left(Y_{i}\left[\begin{array}{ll}
0 & 0 \\
0 & 0
\end{array}\right]-Y_{i}\left[\begin{array}{ll}
1 & 0 \\
0 & 0
\end{array}\right]\right) Q^{1 / 2}\left[\begin{array}{ll}
0 & 0 \\
0 & 0
\end{array}\right] Q^{1 / 2}\left[\begin{array}{ll}
1 & 0 \\
0 & 0
\end{array}\right] \\
& -\left(Y_{i}\left[\begin{array}{ll}
0 & 1 \\
0 & 0
\end{array}\right]-Y_{i}\left[\begin{array}{ll}
1 & 1 \\
0 & 0
\end{array}\right]\right) Q^{1 / 2}\left[\begin{array}{ll}
0 & 1 \\
0 & 0
\end{array}\right] Q^{1 / 2}\left[\begin{array}{ll}
1 & 1 \\
0 & 0
\end{array}\right] \\
& -\left(Y_{i}\left[\begin{array}{ll}
0 & 0 \\
0 & 1
\end{array}\right]-Y_{i}\left[\begin{array}{ll}
1 & 0 \\
0 & 1
\end{array}\right]\right) Q^{1 / 2}\left[\begin{array}{ll}
0 & 0 \\
0 & 1
\end{array}\right] Q^{1 / 2}\left[\begin{array}{ll}
1 & 0 \\
0 & 1
\end{array}\right]=0,
\end{aligned}
$$

which was conjectured in [9] and proved explicitly in [4]. 


\subsection{The terms containg an odd Theta-function.}

Now we prove the vanishing of $V\left[\begin{array}{cc}\alpha_{1}+b_{3} & \alpha_{2} \\ \beta_{1}+b_{4} & \beta_{2}\end{array}\right]$. We have

$$
V\left[\begin{array}{cc}
\alpha_{1}+b_{3} & \alpha_{2} \\
\beta_{1}+b_{4} & \beta_{2}
\end{array}\right] \propto \prod_{f \in F+b_{1}+b_{2}} \Theta^{1 / 2}\left[\begin{array}{cc}
\left(\alpha_{1}+b_{3}\right)(f) & \alpha_{2}(f) \\
\left(\beta_{1}+b_{4}\right)(f) & \beta_{2}(f)
\end{array}\right] .
$$

The strategy to prove the vanishing of the above expression is to show that there always exist at least one odd spin structure for the $\Theta$-function. As we proved in section 2, for any choice of $\alpha_{1}$ and $\beta_{1}$, one can find fermionic field $f$ in $b=F+b_{1}+b_{2}$ such that $\left[\begin{array}{c}\alpha_{1}+b_{3} \\ \beta_{1}+b_{4}\end{array}\right]=\left[\begin{array}{l}1 \\ 1\end{array}\right]$. For such $f$, $\left[\begin{array}{ll}\left(\alpha_{1}+b_{3}\right)(f) & \alpha_{2}(f) \\ \left(\beta_{1}+b_{4}\right)(f) & \beta_{2}(f)\end{array}\right]$ takes the values $\left[\begin{array}{ll}1 & 0 \\ 1 & 0\end{array}\right],\left[\begin{array}{ll}1 & 1 \\ 1 & 0\end{array}\right]$ or $\left[\begin{array}{ll}1 & 0 \\ 1 & 1\end{array}\right]$ for $\left[\begin{array}{l}\alpha_{2} \\ \beta_{2}\end{array}\right]=\left[\begin{array}{l}\emptyset \\ \emptyset\end{array}\right],\left[\begin{array}{l}b \\ \emptyset\end{array}\right]$ or $\left[\begin{array}{l}\emptyset \\ b\end{array}\right]$ respectively. All these are odd spin structures. For $\left[\begin{array}{l}\alpha_{2} \\ \beta_{2}\end{array}\right]=\left[\begin{array}{l}b \\ b\end{array}\right]$, we have

$$
\left[\begin{array}{cc}
\left(\alpha_{1}+b_{3}\right)(f) & \alpha_{2}(f) \\
\left(\beta_{1}+b_{4}\right)(f) & \beta_{2}(f)
\end{array}\right]=\left[\begin{array}{cc}
\left(\alpha_{1}+b_{3}\right)(f) & 1 \\
\left(\beta_{1}+b_{4}\right)(f) & 1
\end{array}\right] .
$$

To have a possibly non-vansihing result, this must be an even spin structure for all $f \in b$. This requires

$$
\left(\alpha_{1}+b_{3}\right)(f)=\left(\beta_{1}+b_{4}\right)(f)=1, \forall f \in b,
$$

In particular this implies

$$
\left(\alpha_{1}+b_{3}\right) \cap(b)=b,
$$

which is not the case for any choice of $\alpha_{1} \in \Xi_{0}^{\prime}=\{\emptyset, b\}$.

\subsection{The last step: the non-vanishing terms}

The last step is to compute $V\left[\begin{array}{cc}\alpha_{1}+b_{3} & \alpha_{2}+b_{4} \\ \beta_{1} & \beta_{2}\end{array}\right]$. First we note the following result:

$$
V\left[\begin{array}{cc}
\alpha_{1}+b_{3} & \alpha_{2}+b_{4} \\
\beta_{1} & \beta_{2}
\end{array}\right]=0, \quad \text { for } \quad \beta_{1}=F+b_{1}+b_{2} \quad \text { or } \quad \beta_{2}=F+b_{1}+b_{2},
$$


which can be proved by following the method of the last paragraph. Now we compute $V\left[\begin{array}{cc}\alpha_{1}+b_{3} & \alpha_{2}+b_{4} \\ \emptyset & \emptyset\end{array}\right]$. For simplicity let us first take $\alpha_{1}=\alpha_{2}=\emptyset$ and we have

$$
\begin{aligned}
& V\left[\begin{array}{cc}
b_{3} & b_{4} \\
\emptyset & \emptyset
\end{array}\right]=\sum_{n_{i}, m_{i}, \tilde{n}_{i}, \tilde{m}_{i}=0}^{1}\langle J(r+) J(r-) \tilde{J}(\bar{s}+) \tilde{J}(\bar{s}+)\rangle_{s(\psi, \tilde{\psi})} \\
& \quad \times C\left[\begin{array}{cc}
b_{3}+n_{1} b_{1}+m_{1} b_{2} & b_{4}+n_{2} b_{1}+m_{2} b_{2} \\
\tilde{n}_{1} b_{1}+\tilde{m}_{1} b_{2} & \tilde{n}_{2} b_{1}+\tilde{m}_{2} b_{2}
\end{array}\right] \\
& \quad \times Q^{1 / 2}\left[\begin{array}{ll}
n_{1} & n_{2} \\
\tilde{n}_{1} & \tilde{n}_{2}
\end{array}\right] Q^{1 / 2}\left[\begin{array}{cc}
n_{1}+1 & n_{2} \\
\tilde{n}_{1} & \tilde{n}_{2}
\end{array}\right] \bar{Q}^{1 / 2}\left[\begin{array}{cc}
m_{1} & m_{2} \\
\tilde{m}_{1} & \tilde{m}_{2}
\end{array}\right] \bar{Q}^{1 / 2}\left[\begin{array}{cc}
m_{1} & m_{2}+1 \\
\tilde{m}_{1} & \tilde{m}_{2}
\end{array}\right] \\
& \quad \times\left|Q\left[\begin{array}{ll}
0 & 0 \\
0 & 0
\end{array}\right] \bar{Q}\left[\begin{array}{ll}
1 & 1 \\
0 & 0
\end{array}\right]\right| Q^{1 / 2}\left[\begin{array}{ll}
0 & 1 \\
0 & 0
\end{array}\right] \bar{Q}^{1 / 2}\left[\begin{array}{ll}
1 & 0 \\
0 & 0
\end{array}\right] .
\end{aligned}
$$

The coeffieints $C$ we needed are given as follows:

$$
\begin{aligned}
& C\left[\begin{array}{cc}
b_{3}+n_{1} b_{1}+m_{1} b_{2} & b_{4}+n_{2} b_{1}+m_{2} b_{2} \\
\tilde{n}_{1} b_{1}+\tilde{m}_{1} b_{2} & \tilde{n}_{2} b_{1}+\tilde{m}_{2} b_{2}
\end{array}\right] \\
& \quad=C\left[\begin{array}{c}
b_{3}+n_{1} b_{1}+m_{1} b_{2} \\
\tilde{n}_{1} b_{1}+\tilde{m}_{1} b_{2}
\end{array}\right] C\left[\begin{array}{c}
b_{4}+n_{2} b_{1}+m_{2} b_{2} \\
\tilde{n}_{2} b_{1}+\tilde{m}_{2} b_{2}
\end{array}\right] \\
& \quad=C\left[\begin{array}{c}
b_{3}+n_{1} b_{1} \\
\tilde{n}_{1} b_{1}
\end{array}\right] C\left[\begin{array}{c}
b_{3}+m_{1} b_{2} \\
\tilde{m}_{1} b_{2}
\end{array}\right] C\left[\begin{array}{c}
b_{4}+n_{2} b_{1} \\
\tilde{n}_{2} b_{1}
\end{array}\right] C\left[\begin{array}{c}
b_{4}+m_{2} b_{2} \\
\tilde{m}_{2} b_{2}
\end{array}\right] .(63)
\end{aligned}
$$

We note that in eq. (62) only $\tilde{n}_{1}=\tilde{m}_{2}=0$ contribute. With this restriction the ambiguity in eq. (63) drops and we have

$$
C\left[\begin{array}{c}
b_{3}+n_{1} b_{1} \\
\tilde{n}_{1} b_{1}
\end{array}\right]=(-1)^{n_{1}}, \quad C\left[\begin{array}{c}
b_{4}+m_{2} b_{2} \\
\tilde{m}_{2} b_{2}
\end{array}\right]=(-1)^{m_{2}} .
$$

and

$$
C\left[\begin{array}{c}
b_{3}+m_{1} b_{2} \\
\tilde{m}_{1} b_{2}
\end{array}\right]=(-1)^{m_{1}}, \quad C\left[\begin{array}{c}
b_{4}+n_{2} b_{1} \\
\tilde{n}_{2} b_{1}
\end{array}\right]=(-1)^{n_{2}} .
$$

We note here that if we have (see eq. (21))

$$
C\left[\begin{array}{l}
b_{1} \\
b_{4}
\end{array}\right]=-1, \quad \text { and } \quad C\left[\begin{array}{l}
b_{2} \\
b_{3}
\end{array}\right]=-1,
$$


eq. (65) would become

$$
C\left[\begin{array}{c}
b_{3}+m_{1} b_{2} \\
\tilde{m}_{1} b_{2}
\end{array}\right]=(-1)^{m_{1}+\tilde{m}_{1}}, \quad C\left[\begin{array}{c}
b_{4}+n_{2} b_{1} \\
\tilde{n}_{2} b_{1}
\end{array}\right]=(-1)^{n_{2}+\tilde{n}_{2}} .
$$

Substituting eqs. (63)-(65) into eq. (62), the left part of spin structure dependent terms are

$$
\begin{aligned}
V_{i} & =\left(Y_{i}\left[\begin{array}{ll}
0 & 0 \\
0 & 0
\end{array}\right]-Y_{i}\left[\begin{array}{ll}
1 & 0 \\
0 & 0
\end{array}\right]\right) Q^{1 / 2}\left[\begin{array}{ll}
0 & 0 \\
0 & 0
\end{array}\right] Q^{1 / 2}\left[\begin{array}{ll}
1 & 0 \\
0 & 0
\end{array}\right] \\
& -\left(Y_{i}\left[\begin{array}{ll}
0 & 1 \\
0 & 0
\end{array}\right]-Y_{i}\left[\begin{array}{ll}
1 & 1 \\
0 & 0
\end{array}\right]\right) Q^{1 / 2}\left[\begin{array}{ll}
0 & 1 \\
0 & 0
\end{array}\right] Q^{1 / 2}\left[\begin{array}{ll}
1 & 1 \\
0 & 0
\end{array}\right] \\
& +\left(Y_{i}\left[\begin{array}{ll}
0 & 0 \\
0 & 1
\end{array}\right]-Y_{i}\left[\begin{array}{ll}
1 & 0 \\
0 & 1
\end{array}\right]\right) Q^{1 / 2}\left[\begin{array}{ll}
0 & 0 \\
0 & 1
\end{array}\right] Q^{1 / 2}\left[\begin{array}{ll}
1 & 0 \\
0 & 1
\end{array}\right] .
\end{aligned}
$$

We draw the attention of the readers to the difference between eq. (56) and eq. (68). It's the minor (but crucial) sign difference on the third line which gives a non-vanishing result. We have

$$
\begin{aligned}
V_{i} & =2\left(Y_{i}\left[\begin{array}{ll}
0 & 0 \\
0 & 1
\end{array}\right]-Y_{i}\left[\begin{array}{ll}
1 & 0 \\
0 & 1
\end{array}\right]\right) Q^{1 / 2}\left[\begin{array}{ll}
0 & 0 \\
0 & 1
\end{array}\right] Q^{1 / 2}\left[\begin{array}{ll}
1 & 0 \\
0 & 1
\end{array}\right] \\
& =\left(a_{2}^{i}-a_{3}^{i}\right) Q^{1 / 2}\left[\begin{array}{ll}
0 & 0 \\
0 & 1
\end{array}\right] Q^{1 / 2}\left[\begin{array}{ll}
1 & 0 \\
0 & 1
\end{array}\right]
\end{aligned}
$$

if we set $r=\infty$ (the left supercurrent insertion points). Similar calculation can be done also for the right part. The result is

$$
\bar{V}_{i}=\left(\bar{a}_{4}^{i}-\bar{a}_{5}^{i}\right) \bar{Q}^{1 / 2}\left[\begin{array}{ll}
0 & 0 \\
1 & 0
\end{array}\right] \bar{Q}^{1 / 2}\left[\begin{array}{ll}
0 & 1 \\
1 & 0
\end{array}\right]
$$

if we set $r=\infty$ (the right supercurrent insertion points).

By combining the left and right part, the result is

$$
V_{i \bar{j}}\left[\begin{array}{cc}
b_{3} & b_{4} \\
\emptyset & \emptyset
\end{array}\right]=a_{23}^{i} \bar{a}_{45}^{j}\left(a_{134526} \bar{a}_{164325}\right)\left|(124 \mid 356)^{2}(135 \mid 246)\right|,
$$

where $a_{i j k l m n} \equiv a_{i j} a_{j k} a_{k l} a_{l m} a_{m n} a_{n i}$ and similarly for $\bar{a}_{i j k l m n}$. Here we have used the explicit expression for $Q$ eq.(53) and droped all other non-essential factors. 
For other $\alpha_{i}$, we can do similar calculation and the results are:

$$
\begin{aligned}
V_{i \bar{j}}\left[\begin{array}{cc}
b+b_{3} & b_{4} \\
\emptyset & \emptyset
\end{array}\right] & =a_{23}^{i} \bar{a}_{45}^{j}\left(a_{124536} \bar{a}_{164235}\right)\left|(134 \mid 256)^{2}(125 \mid 346)\right|, \\
V_{i \bar{j}}\left[\begin{array}{cc}
b_{3} & b+b_{4} \\
\emptyset & \emptyset
\end{array}\right] & =a_{23}^{i} \bar{a}_{45}^{j}\left(a_{135426} \bar{a}_{165324}\right)\left|(125 \mid 346)^{2}(134 \mid 256)\right|, \\
V_{i \bar{j}}\left[\begin{array}{cc}
b+b_{3} & b+b_{4} \\
\emptyset & \emptyset
\end{array}\right] & =a_{23}^{i} \bar{a}_{45}^{j}\left(a_{125436} \bar{a}_{165234}\right)\left|(135 \mid 246)^{2}(124 \mid 356)\right| .
\end{aligned}
$$

From the above results, we observe a general pattern for the non-vanishing amplitude. By comparing eq. (71) with eq. (72) we see that $V_{i \bar{j}}\left[\begin{array}{cc}b+b_{3} & b_{4} \\ \emptyset & \emptyset\end{array}\right]$ can be obtained from $V_{i \bar{j}}\left[\begin{array}{cc}b_{3} & b_{4} \\ \emptyset & \emptyset\end{array}\right]$ by a modular transformation $a_{2} \leftrightarrow a_{3}$. We also note that under this transformation $\bar{a}_{164325}$ changes to $-\bar{a}_{164235}$. There is one other sign change from the left part: $a_{23}^{i} \rightarrow-a_{23}^{i}$. We claim that all the other non-vanishing amplitude can all be obtained from this single $V_{i \bar{j}}\left[\begin{array}{cc}b_{3} & b_{4} \\ \emptyset & \emptyset\end{array}\right]$ non-vanishing expression by doing all the possible different modular transformations. To prove this claim we introduce the following definition:

$$
\begin{aligned}
V_{i \bar{j}}\left(A_{1},\right. & \left.A_{2}, A_{3}, A_{4}, A_{5}, A_{6}\right) \\
\equiv & \left(A_{2}^{i}-A_{5}^{i}\right)\left(\bar{A}_{3}^{j}-\bar{A}_{4}^{j}\right)\left(A_{1} A_{2} A_{3} A_{4} A_{5} A_{6}\right)\left(\bar{A}_{1} \bar{A}_{6} \bar{A}_{3} \bar{A}_{2} \bar{A}_{5} \bar{A}_{4}\right) \\
& \times\left|\left(A_{1} A_{3} A_{5} \mid A_{2} A_{4} A_{6}\right)^{2}\left(A_{1} A_{2} A_{4} \mid A_{3} A_{5} A_{6}\right)\right| .
\end{aligned}
$$

where $\left(A_{1}, A_{2}, A_{3}, A_{4}, A_{5}, A_{6}\right)$ is a permutation of $\left(a_{1}, a_{2}, a_{3}, a_{4}, a_{5}, a_{6}\right)$ and $\left(A_{1} A_{2} A_{3} A_{4} A_{5} A_{6}\right) \equiv\left(A_{1}-A_{2}\right)\left(A_{2}-A_{3}\right)\left(A_{3}-A_{4}\right)\left(A_{4}-A_{5}\right)\left(A_{5}-A_{6}\right)\left(A_{6}-A_{1}\right)$, etc. It is easy to prove the following property of $V_{i}$ :

$$
V_{i \bar{j}}\left(A_{6}, A_{5}, A_{4}, A_{3}, A_{2}, A_{1}\right)=V_{i \bar{j}}\left(A_{1}, A_{2}, A_{3}, A_{4}, A_{5}, A_{6}\right) .
$$

By using this property, the number of different $V_{i \bar{j}}$ is $6 ! / 2=360$. This is exactly the number of non-vanishing $V$ []'s: $90 \times 4=360$ because every $V$ [] from the 6th category (there are 90 different of them in this category) would give 4 non-vanishing terms as we explicitly showed in eqs. (71)-(74). 
By summing over all these permutations $\sigma$ we finally get the following non-vanishing and explicitly modular invariant expression

$$
V_{i \bar{j}}=\sum_{\sigma} V_{i \bar{j}}\left(\sigma\left(a_{1}\right), \sigma\left(a_{2}\right), \sigma\left(a_{3}\right), \sigma\left(a_{4}\right), \sigma\left(a_{5}\right), \sigma\left(a_{6}\right)\right),
$$

which gives contribution to the integrand of the two-loop cosmological constant. We see no reason why a non-vanishng integrand should give a vanishing cosmological constant after the integration over the moduli space.

\section{Appendix A: Analysis of the gravitini content of the model}

Here we show that in the model of eq. (18) there are no gravitini in the free spectrum. The analysis is done by looking at the one-loop partition function. First, note that

$$
\operatorname{Tr}(-)^{\beta} R^{\alpha} N S^{F+\alpha} \sim \prod_{f \in F} \Theta^{1 / 2}\left[\begin{array}{l}
\alpha(f) \\
\beta(f)
\end{array}\right]
$$

with a self-explanatory notation. Therefore the partition function can be written as

$$
Z \sim \sum_{\alpha, \beta} C\left[\begin{array}{l}
\alpha \\
\beta
\end{array}\right] \operatorname{Tr}(-)^{\beta} R^{\alpha} N S^{F+\alpha}
$$

Now by writing $\beta=\tilde{\beta}+n_{i} b_{i}$ with $n_{i}=0,1$ and using eq. (15) and denoting $\delta_{\alpha} \equiv(-)^{\alpha(\psi, \tilde{\psi})}$, we have

$$
Z \sim \sum_{\alpha, \tilde{\beta}} \operatorname{Tr}(-)^{\tilde{\beta}} C\left[\begin{array}{l}
\alpha \\
\tilde{\beta}
\end{array}\right]\left(1+\delta_{\alpha}(-)^{b_{i}} C\left[\begin{array}{l}
\alpha \\
b_{i}
\end{array}\right]\right) R^{\alpha} N S^{F+\alpha}
$$

Of course now $\tilde{\beta}$ runs over the set else than $b_{i}$. We iterate this procedure for other base elements $b_{j}$ until

$$
C\left[\begin{array}{c}
\alpha \\
\tilde{\beta}
\end{array}\right] \rightarrow C\left[\begin{array}{l}
\alpha \\
\emptyset
\end{array}\right]=\delta_{\alpha}
$$


(the last equality follows from eq. (15)), and finally we get the following projections:

$$
Z \sim \sum_{\alpha} \delta_{\alpha} \prod_{i=0}^{N} \frac{1}{2}\left(1+\delta_{\alpha}(-)^{b_{i}} C\left[\begin{array}{l}
\alpha \\
b_{i}
\end{array}\right]\right) R^{\alpha} N S^{F+\alpha}
$$

Now we see that taking $\alpha=b_{1}$ (see eq. (18)) and $b_{i}=b_{2}, b_{3}$ for Susy/NonSusy models we get

$$
\operatorname{Tr} \frac{1}{2}\left(1 \pm(-)^{b_{3}}\right) \times \frac{1}{2}\left(1-(-)^{b_{2}} C\left[\begin{array}{l}
b_{1} \\
b_{2}
\end{array}\right]\right) R^{b_{1}} N S^{F+b_{1}} .
$$

This means that a massless state could be obtained by taking the vacuum in the left sector (Ramond for the fermions in $b_{1}$ thus including the spacetime left fermions and NeveuSchwarz for the other left fermions: thus getting a spacetime spinor) and exciting one NeveuSchwarz lowest mode of the spacetime right fermions (thus a spacetime vector). Because $b_{2}$ contains the spacetime right fermions, all gravitino states are not projected out by the first

projection if $C\left[\begin{array}{l}b_{1} \\ b_{2}\end{array}\right]=1$. On the other, since $b_{3}$ does not contain spacetime right fermions this gravitino state is projected out in the NonSusy model.

\section{Appendix B: The classification of the two-loop terms into modular invariant sets}

Here we organize the various contributions in modular invariant sets, that is we put in the same set contributions related by modular transformations. We denote a one-loop spin structure by

$$
\left[\begin{array}{l}
a \\
b
\end{array}\right]
$$

A two-loops spin structure can be denoted by the tensor product of two one-loop spin structures:

$$
\left[\begin{array}{ll}
a_{1} & a_{2} \\
b_{1} & b_{2}
\end{array}\right] \sim\left[\begin{array}{l}
a_{1} \\
b_{1}
\end{array}\right] \times\left[\begin{array}{l}
a_{2} \\
b_{2}
\end{array}\right]
$$


As we have seen, we have to consider the cases when $a(b)=\alpha+n b_{3,4}(\beta+$ $\left.\tilde{n} b_{3,4}\right)$ where $\alpha, \beta \in \Xi_{0}^{\prime}=\left\{\emptyset, F+b_{1}+b_{2}\right\}$. Let us for short take $\alpha=\beta=\emptyset$. At one-loop the following three are related by modular transformations:

$$
\left[\begin{array}{l}
v \\
\emptyset
\end{array}\right], \quad\left[\begin{array}{l}
\emptyset \\
v
\end{array}\right], \quad\left[\begin{array}{c}
v \\
v+F
\end{array}\right] \sim\left[\begin{array}{l}
v \\
v
\end{array}\right]
$$

(we are interested in the case when $v=b_{3}, b_{4}, b_{3}+b_{4}$ ). Thus we consider one representative say $\left[\begin{array}{l}v \\ \emptyset\end{array}\right]$ for the 3 elements. Similarly the various $\left[\begin{array}{l}v \\ u\end{array}\right]$ are all modular related, for $u \neq v$ and $u, v=b_{3}, b_{4}, b_{3}+b_{4}$, for a total of 6 elements: we take as representative $\left[\begin{array}{l}b_{3} \\ b_{4}\end{array}\right]$.

The two-loops modular transformations (see below) give the relations, for $u \neq v$ and $u, v=b_{3}, b_{4}$ :

$$
\begin{aligned}
& {\left[\begin{array}{l}
v \\
\emptyset
\end{array}\right] \times\left[\begin{array}{l}
v \\
\emptyset
\end{array}\right] \sim\left[\begin{array}{l}
\emptyset \\
\emptyset
\end{array}\right] \times\left[\begin{array}{l}
v \\
\emptyset
\end{array}\right],} \\
& {\left[\begin{array}{l}
v \\
\emptyset
\end{array}\right] \times\left[\begin{array}{c}
v+u \\
\emptyset
\end{array}\right] \sim\left[\begin{array}{l}
v \\
\emptyset
\end{array}\right] \times\left[\begin{array}{l}
u \\
\emptyset
\end{array}\right],} \\
& {\left[\begin{array}{l}
v \\
\emptyset
\end{array}\right] \times\left[\begin{array}{l}
v \\
u
\end{array}\right] \sim\left[\begin{array}{l}
\emptyset \\
\emptyset
\end{array}\right] \times\left[\begin{array}{l}
v \\
u
\end{array}\right],} \\
& {\left[\begin{array}{c}
v+u \\
\emptyset
\end{array}\right] \times\left[\begin{array}{l}
v \\
u
\end{array}\right] \sim\left[\begin{array}{l}
\emptyset \\
\emptyset
\end{array}\right] \times\left[\begin{array}{l}
v \\
u
\end{array}\right],} \\
& {\left[\begin{array}{l}
v \\
u
\end{array}\right] \times\left[\begin{array}{l}
v \\
u
\end{array}\right] \sim\left[\begin{array}{l}
v \\
\emptyset
\end{array}\right] \times\left[\begin{array}{l}
u \\
\emptyset
\end{array}\right] .}
\end{aligned}
$$

In conclusion we get the 6 categories written in Sect.4-Organization of the computation, each category being a set of elements with the given multiplicity. For instance, $\left[\begin{array}{cc}\alpha_{1}+b_{3} & \alpha_{2} \\ \beta_{1} & \beta_{2}\end{array}\right]$ is obtained from

$$
\left[\begin{array}{l}
v \\
\emptyset
\end{array}\right] \times\left[\begin{array}{l}
v \\
\emptyset
\end{array}\right], \quad\left[\begin{array}{l}
\emptyset \\
\emptyset
\end{array}\right] \times\left[\begin{array}{l}
v \\
\emptyset
\end{array}\right], \quad\left[\begin{array}{l}
v \\
\emptyset
\end{array}\right] \times\left[\begin{array}{l}
\emptyset \\
\emptyset
\end{array}\right]
$$

for a total of $3 \times 3+2 \times 3=15$ modular related terms. For the other categories we have a similar counting: $2 \times(6+3 \times(3 \times 6))=120$ terms for the category $5)$ and $6 \times 6+2 \times(3 \times(3 \times 3))=90$ terms for the category 6$)$. 
Finally, the two-loop modular transformation we have used above is:

$$
Q\left[\begin{array}{ll}
a_{1} & a_{2} \\
b_{1} & b_{2}
\end{array}\right] \stackrel{\mathrm{x}}{\longrightarrow} \pm Q\left[\begin{array}{cc}
a_{1} & a_{2} \\
a_{2}+b_{1} & a_{1}+b_{2}
\end{array}\right]
$$

which would give the following modular transformation for $V[]$ :

$$
V\left[\begin{array}{cc}
\alpha_{1} & \alpha_{2} \\
\beta_{1} & \beta_{2}
\end{array}\right] \stackrel{\mathrm{x}}{\longrightarrow} V\left[\begin{array}{cc}
\alpha_{1} & \alpha_{2} \\
\alpha_{2}+\beta_{1} & \alpha_{1}+\beta_{2}
\end{array}\right]
$$

The other generating modular transformations are just one-loop like modular transformations which change only one column of the characteristics of the theta function, i.e. $Q_{s}$. These are denoted as $T_{1,2}$ and $S_{1,2}$ in an obvious notation. The following example demonstrates how we change $V\left[\begin{array}{cc}\alpha_{1}+b_{3} & \alpha_{2}+b_{4} \\ \beta_{1} & \beta_{2}\end{array}\right]$ to $V\left[\begin{array}{cc}\tilde{\alpha}_{1}+b_{4} & \tilde{\alpha}_{2}+b_{3} \\ \tilde{\beta}_{1} & \tilde{\beta}_{2}\end{array}\right]$ by a series of modular transformations:

$$
\begin{aligned}
& V\left[\begin{array}{cc}
\alpha_{1}+b_{3} & \alpha_{2}+b_{4} \\
\beta_{1} & \beta_{2}
\end{array}\right] \stackrel{\mathrm{x}}{\longrightarrow} V\left[\begin{array}{cc}
\alpha_{1}+b_{3} & \alpha_{2}+b_{4} \\
\alpha_{2}+\beta_{1}+b_{4} & \alpha_{1}+\beta_{2}+b_{3}
\end{array}\right] \\
& \stackrel{\mathrm{S}_{1} \mathrm{~S}_{2}}{\longrightarrow} V\left[\begin{array}{cc}
\alpha_{2}+\beta_{1}+b_{4} & \alpha_{1}+\beta_{2}+b_{3} \\
\alpha_{1}+b_{3} & \alpha_{2}+b_{4}
\end{array}\right] \\
& \stackrel{\mathrm{x}}{\longrightarrow} V\left[\begin{array}{cc}
\alpha_{2}+\beta_{1}+b_{4} & \alpha_{1}+\beta_{2}+b_{3} \\
\beta_{2} & \beta_{1}
\end{array}\right] .
\end{aligned}
$$

\section{Appendix C: Two-loop vanishing of the cos- mological constant in supersymmetric string models}

First let us show that $V\left[\begin{array}{ll}\alpha_{1} & \alpha_{2} \\ \beta_{1} & \beta_{2}\end{array}\right]=0$. Because $\alpha_{i}, \beta_{j} \in \Xi_{0}^{\prime}, i, j=1,2$, we have $b_{k} \cap \alpha_{i}=b_{k} \cap \beta_{j}=\emptyset, k=1,2$, and

$$
\begin{aligned}
& V\left[\begin{array}{cc}
\alpha_{1} & \alpha_{2} \\
\beta_{1} & \beta_{2}
\end{array}\right] \propto \sum_{n_{i}, m_{i}, \tilde{n}_{i}, \tilde{m}_{i}=0}^{1} C\left[\begin{array}{cc}
\alpha_{1}+n_{1} b_{1}+m_{1} b_{2} & \alpha_{2}+n_{2} b_{1}+m_{2} b_{2} \\
\beta_{1}+\tilde{n}_{1} b_{1}+\tilde{m}_{1} b_{2} & \beta_{2}+\tilde{n}_{2} b_{1}+\tilde{m}_{2} b_{2}
\end{array}\right] \\
& \quad \times\langle J(r+) J(r-) \tilde{J}(\bar{s}+) \tilde{J}(\bar{s}+)\rangle_{s(\psi, \tilde{\psi})} Q\left[\begin{array}{cc}
n_{1} & n_{2} \\
\tilde{n}_{1} & \tilde{n}_{2}
\end{array}\right] \bar{Q}\left[\begin{array}{cc}
m_{1} & m_{2} \\
\tilde{m}_{1} & \tilde{m}_{2}
\end{array}\right] .
\end{aligned}
$$


Noticing the following result for the coefficients $C$ :

$$
\begin{aligned}
& C\left[\begin{array}{c}
\alpha_{1}+n_{1} b_{1}+m_{1} b_{2} \\
\beta_{1}+\tilde{n}_{1} b_{1}+\tilde{m}_{1} b_{2} b_{2}+\beta_{2}+\tilde{n}_{2} b_{1}+\tilde{m}_{2} b_{2}
\end{array}\right] \\
& \quad=C\left[\begin{array}{c}
\alpha_{1}+n_{1} b_{1}+m_{1} b_{2} \\
\beta_{1}+\tilde{n}_{1} b_{1}+\tilde{m}_{1} b_{2}
\end{array}\right] C\left[\begin{array}{c}
\alpha_{2}+n_{2} b_{1}+m_{2} b_{2} \\
\beta_{2}+\tilde{n}_{2} b_{1}+\tilde{m}_{2} b_{2}
\end{array}\right] \\
& \quad=C\left[\begin{array}{c}
\alpha_{1} \\
\beta_{1}
\end{array}\right] C\left[\begin{array}{c}
\alpha_{2} \\
\beta_{2}
\end{array}\right](-1)^{n_{1}+n_{2}+\tilde{n}_{1}+\tilde{n}_{2}}(-1)^{m_{1}+m_{2}+\tilde{m}_{1}+\tilde{m}_{2}},
\end{aligned}
$$

the summation over the spin structure dependent parts in (96) is factorized into left and right parts. For the left part we have

$$
\begin{gathered}
V_{L}\left[\begin{array}{cc}
\alpha_{1} & \alpha_{2} \\
\beta_{1} & \beta_{2}
\end{array}\right] \propto \sum_{n_{i}, \tilde{n}_{i}=0}^{1}\langle J(r+) J(r-)\rangle_{s\left(\psi, \chi^{I}, y^{I}, \omega^{I}\right)}(-1)^{n_{1}+n_{2}+\tilde{n}_{1}+\tilde{n}_{2}} Q\left[\begin{array}{ll}
n_{1} & n_{2} \\
\tilde{n}_{1} & \tilde{n}_{2}
\end{array}\right] \\
=\sum_{n_{i}, \tilde{n}_{i}=0}^{1}(-1)^{n_{1}+n_{2}+\tilde{n}_{1}+\tilde{n}_{2}}\left(K_{1} Y_{1}\left[\begin{array}{ll}
n_{1} & n_{2} \\
\tilde{n}_{1} & \tilde{n}_{2}
\end{array}\right]+K_{2} Y_{2}\left[\begin{array}{ll}
n_{1} & n_{2} \\
\tilde{n}_{1} & \tilde{n}_{2}
\end{array}\right]\right. \\
\left.\quad-\sum_{I=1}^{6} Y_{1}^{2}\left[\begin{array}{ll}
\alpha_{1}\left(y^{I}\right) & \alpha_{2}\left(y^{I}\right) \\
\beta_{1}\left(y^{I}\right) & \beta_{2}\left(y^{I}\right)
\end{array}\right] Y_{1}\left[\begin{array}{ll}
n_{1} & n_{2} \\
\tilde{n}_{1} & \tilde{n}_{2}
\end{array}\right]\right) Q\left[\begin{array}{ll}
n_{1} & n_{2} \\
\tilde{n}_{1} & \tilde{n}_{2}
\end{array}\right] \\
=0,
\end{gathered}
$$

which was proved in [3].

The second is to prove $V\left[\begin{array}{cc}\alpha_{1}+b_{3} & \alpha_{2} \\ \beta_{1} & \beta_{2}\end{array}\right]=0$. We have

$$
\begin{aligned}
V & {\left[\begin{array}{cc}
\alpha_{1}+b_{3} & \alpha_{2} \\
\beta_{1} & \beta_{2}
\end{array}\right] \propto \sum_{n_{i}, m_{i}, \tilde{n}_{i}, \tilde{m}_{i}=0}^{1}\langle J(r+) J(r-) \tilde{J}(\bar{s}+) \tilde{J}(\bar{s}+)\rangle_{s(\psi, \tilde{\psi})} } \\
& \times C\left[\begin{array}{cc}
\alpha_{1}+b_{3}+n_{1} b_{1}+m_{1} b_{2} & \alpha_{2}+n_{2} b_{1}+m_{2} b_{2} \\
\beta_{1}+\tilde{n}_{1} b_{1}+\tilde{m}_{1} b_{2} & \beta_{2}+\tilde{n}_{2} b_{1}+\tilde{m}_{2} b_{2}
\end{array}\right] \\
& \times Q^{1 / 2}\left[\begin{array}{cc}
n_{1} & n_{2} \\
\tilde{n}_{1} & \tilde{n}_{2}
\end{array}\right] Q^{1 / 2}\left[\begin{array}{cc}
n_{1}+1 & n_{2} \\
\tilde{n}_{1} & \tilde{n}_{2}
\end{array}\right] \bar{Q}\left[\begin{array}{cc}
m_{1} & m_{2} \\
\tilde{m}_{1} & \tilde{m}_{2}
\end{array}\right] .
\end{aligned}
$$

Now we need the following coefficient:

$$
C\left[\begin{array}{cc}
\alpha_{1}+b_{3}+n_{1} b_{1}+m_{1} b_{2} & \alpha_{2}+n_{2} b_{1}+m_{2} b_{2} \\
\beta_{1}+\tilde{n}_{1} b_{1}+\tilde{m}_{1} b_{2} & \beta_{2}+\tilde{n}_{2} b_{1}+\tilde{m}_{2} b_{2}
\end{array}\right]
$$




$$
\begin{aligned}
& =C\left[\begin{array}{c}
\alpha_{1}+b_{3}+n_{1} b_{1}+m_{1} b_{2} \\
\beta_{1}+\tilde{n}_{1} b_{1}+\tilde{m}_{1} b_{2}
\end{array}\right] C\left[\begin{array}{c}
\alpha_{2}+n_{2} b_{1}+m_{2} b_{2} \\
\beta_{2}+\tilde{n}_{2} b_{1}+\tilde{m}_{2} b_{2}
\end{array}\right] \\
& =(-1)^{n_{2}+\tilde{n}_{2}+m_{2}+\tilde{m}_{2}} C\left[\begin{array}{c}
\alpha_{1}+b_{3} \\
\beta_{1}
\end{array}\right] C\left[\begin{array}{c}
\alpha_{2} \\
\beta_{2}
\end{array}\right] \\
& \quad \times C\left[\begin{array}{c}
b_{3}+n_{1} b_{1} \\
\tilde{n}_{1} b_{1}
\end{array}\right] C\left[\begin{array}{c}
b_{3}+m_{1} b_{2} \\
\tilde{m}_{1} b_{2}
\end{array}\right] .
\end{aligned}
$$

Again we find a splitting of the left and right parts in eq. (99). For the left part we have

$$
\begin{gathered}
V_{L}\left[\begin{array}{cc}
\alpha_{1}+b_{3} & \alpha_{2} \\
\beta_{1} & \beta_{2}
\end{array}\right] \propto \sum_{n_{i}, \tilde{n}_{i}=0}^{1}(-1)^{n_{2}+\tilde{n}_{2}}\langle J(r+) J(r-)\rangle_{s\left(\psi, \chi^{I}, y^{I}, \omega^{I}\right)} \\
\quad \times C\left[\begin{array}{c}
b_{3}+n_{1} b_{1} \\
\tilde{n}_{1} b_{1}
\end{array}\right] Q^{1 / 2}\left[\begin{array}{cc}
n_{1} & n_{2} \\
\tilde{n}_{1} & \tilde{n}_{2}
\end{array}\right] Q^{1 / 2}\left[\begin{array}{cc}
n_{1}+1 & n_{2} \\
\tilde{n}_{1} & \tilde{n}_{2}
\end{array}\right] .
\end{gathered}
$$

In the above there appears two different spin structures in $Q$. These two spin structures should both be even in order to have a possibly non-vanishing result. This requires $\tilde{n}_{1}=0$ and we have

$$
\begin{aligned}
& V_{L}\left[\begin{array}{cc}
\alpha_{1}+b_{3} & \alpha_{2} \\
\beta_{1} & \beta_{2}
\end{array}\right] \propto \sum_{n_{1}, n_{2}, \tilde{n}_{2}=0}^{1}(-1)^{n_{1}+n_{2}+\tilde{n}_{2}}\langle J(r+) J(r-)\rangle_{s\left(\psi, \chi^{I}, y^{I}, \omega^{I}\right)} \\
& \times Q^{1 / 2}\left[\begin{array}{cc}
n_{1} & n_{2} \\
0 & \tilde{n}_{2}
\end{array}\right] Q^{1 / 2}\left[\begin{array}{cc}
n_{1}+1 & n_{2} \\
0 & \tilde{n}_{2}
\end{array}\right] \\
& =\sum_{n_{1}, n_{2}, \tilde{n}_{2}=0}^{1}(-1)^{n_{1}+n_{2}+\tilde{n}_{2}}\left(K_{1} Y_{1}\left[\begin{array}{cc}
n_{1} & n_{2} \\
0 & \tilde{n}_{2}
\end{array}\right]+K_{2} Y_{2}\left[\begin{array}{cc}
n_{1} & n_{2} \\
0 & \tilde{n}_{2}
\end{array}\right]\right. \\
& -\sum_{I=1}^{6} Y_{1}\left[\begin{array}{cc}
\left(\alpha_{1}+b_{3}\right)\left(y^{I}\right) & \alpha_{2}\left(y^{I}\right) \\
\beta_{1}\left(y^{I}\right) & \beta_{2}\left(y^{I}\right)
\end{array}\right] Y_{1}\left[\begin{array}{cc}
\left(\alpha_{1}+b_{3}\right)\left(\omega^{I}\right) & \alpha_{2}\left(\omega^{I}\right) \\
\beta_{1}\left(\omega^{I}\right) & \beta_{2}\left(\omega^{I}\right)
\end{array}\right] \\
& \left.\times Y_{1}\left[\begin{array}{cc}
n_{1} & n_{2} \\
0 & \tilde{n}_{2}
\end{array}\right]\right) Q^{1 / 2}\left[\begin{array}{cc}
n_{1} & n_{2} \\
0 & \tilde{n}_{2}
\end{array}\right] Q^{1 / 2}\left[\begin{array}{cc}
n_{1}+1 & n_{2} \\
0 & \tilde{n}_{2}
\end{array}\right] \\
& =0 \text {, }
\end{aligned}
$$

by making use of the following identities $(i=1,2)$ :

$$
\left(Y_{i}\left[\begin{array}{ll}
0 & 0 \\
0 & 0
\end{array}\right]-Y_{i}\left[\begin{array}{ll}
1 & 0 \\
0 & 0
\end{array}\right]\right) Q^{1 / 2}\left[\begin{array}{ll}
0 & 0 \\
0 & 0
\end{array}\right] Q^{1 / 2}\left[\begin{array}{ll}
1 & 0 \\
0 & 0
\end{array}\right]
$$




$$
\begin{aligned}
& -\left(Y_{i}\left[\begin{array}{ll}
0 & 1 \\
0 & 0
\end{array}\right]-Y_{i}\left[\begin{array}{ll}
1 & 1 \\
0 & 0
\end{array}\right]\right) Q^{1 / 2}\left[\begin{array}{ll}
0 & 1 \\
0 & 0
\end{array}\right] Q^{1 / 2}\left[\begin{array}{ll}
1 & 1 \\
0 & 0
\end{array}\right] \\
& -\left(Y_{i}\left[\begin{array}{ll}
0 & 0 \\
0 & 1
\end{array}\right]-Y_{i}\left[\begin{array}{ll}
1 & 0 \\
0 & 1
\end{array}\right]\right) Q^{1 / 2}\left[\begin{array}{ll}
0 & 0 \\
0 & 1
\end{array}\right] Q^{1 / 2}\left[\begin{array}{ll}
1 & 0 \\
0 & 1
\end{array}\right]=0,(103
\end{aligned}
$$

which was proved explicitly in 4 . Similar method can be used to prove the vanishing of all $V$ []'s in the 3rd and 4th categories.

\section{Acknowledgments}

We would like to tkank E. Gava, G. Ferretti and K.S. Narain for helpful discussions. C.-J. Zhu is supported in part by funds from National Natural Science Foundation of China and Pandeng Project. He would also like to thank the hospitality of SISSA where most of the work was done.

\section{References}

[1] M. B. Green, J.H. Schwarz and E. Witten, Superstring Theory, 2 vols., Cambridge University Press, Cambridge, 1987.

[2] J. Polchinski, String Theory, 2 vols., Cambridge University Press, Cambridge, 1998.

[3] E. Gava, R. Iengo and G.M. Sotkov, Phys. Lett. B207 (1988) 283.

[4] R. Iengo, G.M. Sotkov and C.-J. Zhu, Two-loop Vacuum Amplitude in Four-dimensional Heterotic String Models, Phys. Lett. B211 (1988) 425.

[5] S. Kachru and E. Silverstein, Self-Dual Nonsupersymmtric Type II String Compactifications, hep-th/9808056.

[6] G. Shiu and S.-H.H. Tye, Bose-Fermi degeneracy and duality in nonsupersymmetric strings, Nucl. Phys. B542 (1999) 45, hep-th/9808095.

[7] S. Kachru and E. Silverstein, On Vanishing Two Loop Cosmological Constants in Nonsupersymmetric Strings, hep-th/9810129. 
[8] E. Verlinde, and H. Verlinde, Superstring Perturbation Theory, in Superstrings'88, M.Green et al. Eds., World Scientific Publ. Co. Singapore, 1989, p. 222-240.

[9] D. Arnaudon, C.P. Bachas, V. Rivasseau and P. Végreville, On the Vanishing of the Cosmological Constant in Four-Dimensional Superstring Models, Phys. Lett. B195 (1987) 167-176.

[10] I. Antoniadis, C. Bachas and C. Kounnas, Four-Dimensional Superstrings, Nucl. Phys. B289 (1987) 87-108.

[11] H. Kawai, D. C. Lewellen and S.-H. H. Tye, Nucl. Phys. B288 (1987) 1; H. Kawai, D. C. Lewellen, J. A. Schwartz and S.-H. H. Tye, Nucl. Phys. B299 (1988) 431.

[12] I. Antoniadis, C. Bachas, C. Kounnas and P. Windey, Supersymmetry Among Free Fermions and Superstrings, Phys. Lett. 171B (1986) 5156.

[13] E. Verlinde and H. Verlinde, Multiloop Calculations in Covariant Superstring Theory, Phys. Lett. B192 (1987) 95.

[14] J. Fay, Theta Function on Riemann Surfaces, Lecture Notes in Mathematics, Vol. 352, Springer, Berlin, 1973. 